\title{
Metformin Suppresses Monocyte Immunometabolic Activation by SARS-CoV-2 and Spike Protein Subunit 1
}

Theodore J. Cory ${ }^{1}$, Russell S. Emmons ${ }^{2}$, Johnathan R. Yarbro ${ }^{2,3}$, Kierstin L. Davis ${ }^{2}$, Brandt D. Pence ${ }^{2,4, *}$

${ }^{1}$ Department of Clinical Pharmacy and Translational Science, College of Pharmacy, University of Tennessee Health Science Center, Memphis, TN 38163

${ }^{2}$ College of Health Sciences, University of Memphis, Memphis, TN 38152

${ }^{3}$ Department of Medicine, University of Tennessee Health Science Center, Memphis, TN 38163

${ }^{4}$ Center for Nutraceutical and Dietary Supplement Research, University of Memphis, Memphis, TN 38152

* Address correspondence to:

Brandt Pence, PhD

University of Memphis

304 Fieldhouse

495 Zach H. Curlin St.

Memphis, TN 38152

bdpence@memphis.edu 


\section{Abstract}

A hallmark of COVID-19 is a hyperinflammatory state that is associated with severity. Various antiinflammatory therapeutics have shown mixed efficacy in treating COVID-19, and the mechanisms by which hyperinflammation occurs are not well understood. Previous research indicated that monocytes, a key innate immune cell, undergo metabolic reprogramming and produce inflammatory cytokines when stimulated with SARS-CoV-2. We hypothesized that binding by the viral spike protein mediates this effect, and that drugs which regulate immunometabolism could inhibit the inflammatory response in monocytes. Monocytes stimulated with recombinant SARS-CoV-2 spike protein subunit 1 showed a dose-dependent increase in glycolytic metabolism that was associated with production of proinflammatory cytokines including interleukin- 6 and tumor necrosis factor- $\alpha$. This response was dependent on hypoxia-inducible factor-1 $\alpha$, as chetomin inhibited glycolysis and cytokine production. Inhibition of glycolytic metabolism by 2-deoxyglucose (2-DG) or glucose deprivation also inhibited the glycolytic response, and 2-DG strongly suppressed cytokine production. Glucose-deprived monocytes rescued cytokine production by upregulating oxidative phosphorylation, an effect which was not present in 2-DG-treated monocytes due to the known effect of 2-DG on suppressing mitochondrial metabolism. Finally, pre-treatment of monocytes with metformin strongly suppressed spike protein-mediated cytokine production in monocytes, and abrogated glycolytic and mitochondrial metabolism. Likewise, metformin pre-treatment blocked cytokine induction by SARS-CoV-2 strain WA1/2020 in direct infection experiments in monocytes. In summary, the SARS-CoV-2 spike protein induces a pro-inflammatory immunometabolic response in monocytes that can be suppressed by metformin, and metformin likewise suppresses inflammatory responses to live SARS-CoV-2. This has potential implications for the treatment of hyperinflammation during COVID-19. 


\section{Introduction}

The ongoing coronavirus disease 2019 (COVID-19) pandemic has presently more than 3 million lives worldwide as of mid-April $2021^{1}$. COVID-19 is caused by a novel highly pathogenic coronavirus classified as severe acute respiratory syndrome coronavirus-2 (SARS-CoV-2) ${ }^{2}$. A hallmark of severe COVID-19 is hyperinflammation ${ }^{3}$, although cytokine expression patterns in individuals are diverse, leading to controversy over classification of COVID-19 related inflammation as cytokine storm, macrophage activation syndrome, multisystem inflammatory syndrome, etc. Regardless, inflammatory cytokines appear to play a principal role in mediating COVID-19 symptoms, therefore therapies which target these responses are paramount to treating severe COVID-19. As such, a fuller understanding of the cellular and molecular mechanisms mediating hypercytokinemia during SARS-CoV-2 infection is necessary.

Mononuclear phagocytes such as monocytes and macrophages are key constituents of the innate immune system, and produce pro-inflammatory cytokines during viral infection ${ }^{4-7}$. Monocyte and monocyte-derived macrophage infiltration into the lungs has been linked to severe COVID-19 in single cell RNA sequencing studies ${ }^{8-11}$ and postmortem analyses ${ }^{12-15}$ in human patients, as well as during experimental infections in animal models including mice ${ }^{16,17}$, hamsters ${ }^{18}$, and various nonhuman primates ${ }^{19-23}$. Monocytes in individuals infected with SARS-CoV-2 display phenotypic changes associated with hyperinflammation, including reduced HLA-DR expression ${ }^{24-26}$, increased CD16 expression ${ }^{25,27-29}$, and increased cytokine production ${ }^{30-33}$. Both monocytes ${ }^{34-36}$ and monocyte-derived macrophages ${ }^{37,38}$ also produce pro-inflammatory cytokines under direct infection with SARS-CoV-2, although infection at least in macrophages appears to be abortive ${ }^{37,38}$.

The past decade has seen an explosion in scientific interest in the regulation of immune cell activation and function by metabolic reprogramming. Under pro-inflammatory conditions, immune cells - including myeloid cells - generally undergo a switch to aerobic glycolysis which provides ATP sufficient 
to support cellular functions which propagate pro-inflammatory and anti-pathogen host responses ${ }^{39}$.

Recently, Codo et al. demonstrated pro-inflammatory glycolytic reprogramming in monocytes infected with SARS-CoV-2 ${ }^{34}$, and SARS-CoV-2 also appears to alter monocyte lipid metabolism to promote lipid droplet formation which is associated with pro-inflammatory cytokine production ${ }^{35}$.

SARS-CoV-2 therefore appears to reprogram metabolism in monocytes, but the viral factors which mediate these responses are unclear. Research in the 2003 epidemic SARS-CoV-1 suggested that the viral spike protein could mediate pro-inflammatory activation in macrophages ${ }^{40,41}$, and recent evidence suggests the spike protein of SARS-CoV-2 also activates inflammatory responses in macrophages and monocytes both in vitro and in vivo ${ }^{42,43}$. Given this, we hypothesized that spike protein binding to monocytes mediates glycolytic reprogramming to promote pro-inflammatory responses of these cells to SARS-CoV-2. Our results herein support this hypothesis, and we additionally report outcomes from experiments aimed at evaluating the responsible cellular signaling mechanisms, as well as potential pharmaceutical strategies for inhibiting these responses.

\section{Results}

Spike protein subunit 1 reprograms metabolism and promotes inflammatory responses

Recently it was demonstrated that SARS-CoV-2 promotes metabolic reprogramming in monocytes during infection ${ }^{34,35}$. Research in SARS-CoV suggested that the viral spike protein induces inflammatory responses in macrophages ${ }^{40,41}$, and this has recently been replicated using spike protein from SARS-CoV-2 ${ }^{42,43}$. Likewise, spike protein binding to C-type lectins has recently been shown to mediate pro-inflammatory processes in myeloid cells ${ }^{44,45}$. Therefore, we hypothesized that the SARSCoV-2 spike protein mediates a pro-inflammatory metabolic reprogramming in monocytes which could be a basis for hypercytokinemia. Stimulation of isolated human classical monocytes with recombinant spike protein subunit 1 (S1) from SARS-CoV-2 induced glycolytic activation (Figure 1A) and suppressed 
oxidative phosphorylation (OXPHOS, Figure 1C) in a dose-dependent manner. The effect of S1 dose was significant for both extracellular acidification rate $\left(F_{2,14}=72.44, p<0.0001\right.$, Figure $\left.1 B\right)$ and oxygen consumption rate $\left(F_{2,14}=5.785, p=0.0147\right.$, Figure $\left.1 D\right)$ as measured by quantification of area under the response curve.

Additionally, recombinant S1 treatment caused a dose-dependent increase transcription of proinflammatory cytokines (Figure $1 \mathrm{E})$ including $I L 1 B\left(\mathrm{~F}_{2,14}=50.98, \mathrm{p}<0.001\right), \operatorname{lL} 6\left(\right.$ Friedman $\chi_{(\mathrm{df}=2)}^{2}=16$, $\mathrm{p}<0.001), \operatorname{CXCL8}\left(\mathrm{F}_{2,14}=38.19, \mathrm{p}<0.001\right)$, and $\operatorname{TNF}\left(\mathrm{F}_{2,14}=28.41, \mathrm{p}<0.001\right)$ as measured by $\mathrm{qPCR}$. These cytokines have been implicated in the pathogenesis of SARS-CoV-2 and in COVID-19-related hypercytokinemia in several studies ${ }^{25,46-51}$. To confirm that increased transcription resulted in increased protein expression, we evaluated protein concentrations of key cytokines in the supernatant of S1stimulated monocytes by enzyme-linked immunosorbent assay (ELISA) (Figure 1F). S1 increased protein expression of interleukin (IL)-6 (Friedman $\left.\chi_{(d f=2)}^{2}=16, p<0.001\right)$ and tumor necrosis factor (TNF)- $\alpha$ $\left(F_{2,14}=37.73, p<0.001\right)$ in a dose-dependent manner.

\section{Glycolytic response to spike protein is dependent on HIF-1 $\alpha$}

Hypoxia inducible factor (HIF)-1 $\alpha$ was demonstrated nearly 20 years ago to mediate proinflammatory responses in myeloid cells ${ }^{52}$, and has more recently been shown to regulate glycolytic activation in monocytes, macrophages, and other immune cells ${ }^{53-55}$. SARS-CoV-2 activates HIF-1 $\alpha$ mediated glycolysis in monocytes ${ }^{34}$, so we reasoned that this was a likely downstream mechanism by which the viral spike protein causes this similar glycolytic reprogramming in our experiments. As above, treatment of monocytes with S1 activated glycolysis, and this effect was abrogated by pre-treatment with chetomin (Figure $2 A / B, F_{2,12}=42.43, p<0.001$ ), which disrupts the interaction between HIF-1 $\alpha$ and p300 to block the effects of the former ${ }^{56}$. Pre-treatment with chetomin also strongly suppressed the cytokine response due to $\mathrm{S} 1$ treatment (Figure $2 \mathrm{C})$, including blunting transcription of $I L 1 B\left(\mathrm{~F}_{2,12}=27.35\right.$, $\mathrm{p}<0.001)$, IL6 $\left(\mathrm{F}_{2,12}=16.11, \mathrm{p}<0.001\right), \operatorname{CXCL8}\left(\mathrm{F}_{2,12}=25.54, \mathrm{p}<0.001\right)$, and $\operatorname{TNF}\left(\mathrm{F}_{2,12}=29.04, \mathrm{p}<0.001\right)$. As 
such, HIF-1 $\alpha$ appears to be a master regulator of both glycolytic reprogramming and inflammatory activation of monocytes under S1 stimulation.

\section{Suppression of glycolysis alters inflammatory responses to spike protein}

To determine whether metabolic reprogramming is responsible for altered cytokine responses to S1, we suppressed glycolytic responses during S1 treatment using 2-deoxyglucose (2-DG) pretreatment. Treatment of monocytes with 2-DG ablated monocyte glycolytic responses to S1 stimulation (Figure $3 \mathrm{~A}$ ) which was significant by comparison of area under the response curve $\left(\mathrm{t}_{6}=-\right.$ 10.867, $\mathrm{p}<0.0001$, Figure 3B). However, 2-DG also suppressed mitochondrial function in these cells (Figure 2C), though this was non-significant by area under the oxygen consumption $\left(t_{6}=-2.2284\right.$, $p=0.0674$, Figure 3D). This effect has been noted previously during responses to LPS $^{57}$. Anticipating this, we also included a condition where monocytes were cultured under glucose deprivation, as a second method of suppressing glycolytic activation. We noted a similar ablation of glycolytic responses to S1 using this strategy (Figure 3A) which was significant by area under the curve analysis $\left(\mathrm{t}_{6}=-14.045\right.$, $\mathrm{p}<0.0001$, Figure 3B). However, glucose deprivation caused an increase in oxygen consumption after S1 treatment (Figure $3 C$ ) which was significant compared to media- $\left(t_{6}=4.6618, p=0.0069\right)$ or $2-D G\left(t_{6}=-\right.$ 15.607, $\mathrm{p}<0.001$ ) pretreated monocytes (Figure 3D).

Pre-treatment of monocytes with 2-DG also strongly inhibited cytokine expression compared to cells treated with S1(Figure $3 E)$, including transcription of $\operatorname{IL1B}(W=0, \mathrm{p}=0.0313), \operatorname{IL6}\left(\mathrm{t}_{6}=-5.912\right.$, $\mathrm{p}=0.0021), \operatorname{CXCL8}(W=0, \mathrm{p}=0.0313)$, and $\operatorname{TNF}(W=0, \mathrm{p}=0.0313)$. However, glucose deprived monocytes generally maintained their ability to transcribe pro-inflammatory cytokines in response to $\mathrm{S} 1$, with only IL1B expression showing a modest $25.1 \%$ reduction in glucose deprived compared to S1-treated monocytes (Figure 3E, $W=0, p=0.0313$ ). Monocytes appear to utilize fatty acid oxidation to compensate for loss of glycolysis during cytokine responses as has been previously demonstrated with LPS ${ }^{57-60}$, and 
therefore the 2-DG-mediated suppression of S1-induced inflammation is likely due to its ability to suppress both glycolysis and mitochondrial metabolism in concert.

AMPK controls the OXPHOS response to spike protein in the absence of glucose

Activation of OXPHOS appeared to compensate for loss of glycolysis under glucose deprivation conditions in S1-treated monocytes. Therefore, we examined the potential role of AMP-activated protein kinase (AMPK) in mediating this response. Compound C (dorsomorphin) is a selective inhibitor of AMPK ${ }^{61}$, therefore we examined whether compound $\mathrm{C}$ would block OXPHOS activation by $\mathrm{S} 1$ in glucose deprived monocytes. Pre-treatment with compound C slightly reduced ECAR in glucose deprived monocytes compared to media-treated glucose deprived monocytes (Figure 4A/B), although this small effect is likely not biologically important. Compound C pre-treatment inhibited the OXPHOS response to S1 during glucose deprivation, but compound C-treated monocytes did not demonstrate suppressed OXPHOS as compared to S1-stimulated monocytes treated in the presence of glucose (Figure 4C/D, $\left.F_{2,12}=25.81, p<0.001\right)$. Interestingly, compound $C$ pre-treatment had a variable effect on cytokine gene expression, with only TNF expression significantly suppressed in glucose deprived monocytes treated with compound $C$ compared to media (Figure 4E, Friedman $\left.\chi_{(d f=2)}^{2}=10.571, p=0.005\right)$. Expression of $I L 1 B$, IL6, and $C X C L 8$ were not significantly altered by compound $C$ treatment, although $C X C L 8$ showed a trend toward increased expression in glucose deprived monocytes treated with compound C compared to media $(p=0.0603)$.

\section{Metformin abrogates inflammatory response to spike protein}

The small molecule compounds chetomin and 2-deoxyglucose inhibited immunometabolic activation in monocytes, suggesting a potential strategy for treating hypercytokinemia during COVID-19. However, chetomin is not approved for use in humans, although it has shown efficacy in vivo in animal models ${ }^{56}$. Additionally, 2-DG has poor efficacy in humans due to rapid metabolism and limited bioavailability ${ }^{62}$. Therefore, we investigated the ability of the common diabetes and geroprotector drug 
metformin to inhibit cytokine production in S1-stimulated monocytes. Metformin activates AMPK ${ }^{61}$ and (independently of AMPK) opposes the action of HIF-1 $\alpha^{63,64}$, and additionally inhibits mitochondrial metabolism through blocking complex I of the electron transport chain ${ }^{65,66}$, thus we hypothesized that it would have a qualitatively similar effect to 2-DG in inhibiting cytokine production through dual inhibition of glycolysis and OXPHOS.

Pre-treatment with metformin abrogated the glycolytic response to S1 in monocytes (Figure $5 A / B, F_{2,12}=60.05, p<0.001$ ) and strongly inhibited cellular respiration (Figure $5 C / D$, Friedman $\chi^{2}$ $(d f=2)=12.286, p=0.0021)$ in Seahorse assays. Likewise, metformin pre-treatment suppressed cytokine responses to $S 1$ treatment in monocytes (Figure $5 E)$, including $I L 1 B\left(\right.$ Friedman $\left.\chi_{(d f=2)}^{2}=12.286, p=0.0021\right)$,

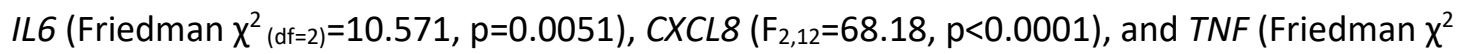
$(d f=2)=12.286, p=0.0021)$.

\section{Metformin abrogates IL-6 production in virus-stimulated monocytes}

Recent evidence suggests that myeloid cells recognize SARS-CoV-2 spike protein through C-type lectins ${ }^{44,45}$. However, the SARS-CoV-2 virion also contains additional immunoregulatory and proinflammatory proteins ${ }^{67,68}$, therefore we examined the ability of metformin to block cytokine responses to live SARS-CoV-2. Monocytes treated for $24 \mathrm{hr}$ with SARS-CoV-2 increased expression of IL-6 protein, and this was suppressed by metformin pre-treatment (Figure $6, F_{2,14}=11.48, p=0.0011$ ), suggesting that the anti-inflammatory effect of metformin is not specific to spike protein-stimulated cytokine responses in monocytes.

\section{Discussion}

The present study resulted in several advances of major importance for the understanding of SARS-CoV-2 innate immune responses. First, we report here that monocytes treated with recombinant spike protein subunit 1 from the current pandemic SARS-CoV-2 undergo a dose-dependent increase in 
glycolysis which is controlled by HIF-1 $\alpha$ and mediates the production of pro-inflammatory cytokines.

These data suggest an initial signaling event which precipitates changes in glucose and lipid metabolism during SARS-CoV-2 infection in monocytes which have been previously reported to be linked to inflammatory activation ${ }^{34,35}$. Monocyte and monocyte-derived macrophages are substantially enriched in the lungs of SARS-CoV-2-infected individuals with severe COVID-19 ${ }^{8,9,12-15}$ and respond to experimental viral infection by producing pro-inflammatory cytokines ${ }^{34-38}$, therefore these results reflect a potential mechanism by which hypercytokinemia occurs during the early innate immune response to SARS-CoV-2.

Importantly, the available evidence suggests that infection of monocytes/macrophages by SARSCoV-2 is abortive ${ }^{37,38,69}$, thus recognition of SARS-CoV-2 structural proteins or genomic material is the likely mechanism by which direct infection precipitates inflammatory responses in this cell type. Our data suggest the spike protein is one such determinant, although we cannot conclude it is the only such mechanism given that recent reports have demonstrated inflammatory responses in macrophages treated with the SARS-CoV-2 envelope protein ${ }^{67}$. It is also possible, however, that direct viral binding to monocytes is not the only way in which these cells can be exposed to the viral spike protein. Recent evidence suggests that vaccine antigens including S1 are released into the circulation following vaccination ${ }^{70}$, and this represents a potential pro-inflammatory stimulus for monocytes. Monocyte/macrophage recognition of S1 may also contribute to the local (muscle) inflammatory response during vaccination. Additionally, the viral spike protein undergoes cleavage by furin during binding to ACE2 ${ }^{71}$, and it has been suggested that this could lead to release of the S1 subunit ${ }^{72}$, although to date this is speculative.

ACE2 has limited expression on immune cells including monocytes and macrophages ${ }^{73}$, which has called into question whether they can directly recognize SARS-CoV-2. In this study we did not identify the mechanism for monocyte recognition of $\mathrm{S} 1$, but several recent papers have shed light on 
this. Two reports recently demonstrated spike binding to C-type lectin receptors ${ }^{44,45}$ which mediates pro-inflammatory signaling in myeloid cells. Likewise, monocytes and macrophages express high levels of $\mathrm{CD} 147^{73}$, and this receptor has been shown to recognize spike protein and contribute to activation of T cells $^{74}$. Monocytes therefore have multiple methods of recognizing $\mathrm{S} 1$, and the receptor(s) responsible for signaling to induce immunometabolic activation deserve further investigation.

The second major advance in this study is the identification of metformin as a potential immunometabolic regulator of inflammatory responses to SARS-CoV-2. Small molecule inhibitors of HIF$1 \alpha$ (chetomin) and glucose metabolism (2-deoxyglucose) blocked cytokine production in S1-treated monocytes, suggesting that interfering with downstream signaling pathways activated by spike protein binding is a potential therapeutic strategy to target inflammation during COVID-19. As these compounds are not approved for human use or have low efficacy in humans as described above, we evaluated the ability of metformin to suppress glycolytic reprogramming and cytokine production in S1-stimulated monocytes. Metformin reduced cytokine production and strongly inhibited both glycolysis and cellular respiration in culture, suggesting it as a potential treatment for hyperinflammation during COVID-19. Further, metformin blocked IL-6 production in monocytes infected with live SARS-CoV-2, suggesting this effect is not limited to artificial stimulation conditions with purified recombinant protein.

Metformin is extremely inexpensive compared to many pharmaceuticals, with an estimated manufacturing cost under 10 USD per kg for the active ingredient ${ }^{75}$ and a monthly wholesale cost as low as 25 USD $^{76}$. Metformin has been previously noted as a treatment for non-COVID acute respiratory distress syndrome ${ }^{77}$ and is a potent suppressor of immune activation of monocytes and macrophages by other molecules including LPS ${ }^{78-80}$. Additionally, several epidemiological studies have noted decreased mortality ${ }^{81-85}$ and inflammation ${ }^{86,87}$ in COVID-19 patients who were taking metformin prior to diagnosis. Therefore, given these observations and its low cost, excellent safety profile, wide availability, and efficacy in inhibiting inflammatory responses to S1 in vitro, metformin is a promising 
candidate for further exploration as a COVID-19 therapeutic. Our study is limited to a single in vitro measure of metformin as a therapeutic for COVID-19, so a great deal of further study is necessary in order to establish this drug as a viable treatment.

\section{Conclusion}

In summary, we demonstrate here that the spike protein subunit 1 from SARS-CoV-2 causes activation of HIF-1 $\alpha$ dependent glycolysis and inflammatory cytokine production in monocytes which can be suppressed by treatment with the diabetes drug metformin. These experiments detail a mechanism by which SARS-CoV-2 mediates metabolic reprogramming previously described in human monocytes, and additionally provides a potential mechanism for the observation that metformin is protective against mortality in COVID-19 patients. Continued research in this area has the potential to define therapeutic strategies and additional molecular targets for the treatment of COVID-19-associated hyperinflammation.

\section{Methods}

\section{Subjects}

Healthy 18-35-year-old subjects ( $\mathrm{N}=14)$ were recruited without respect to sex or race.

Participants reported to the laboratory approximately every two weeks for blood collection, and 8-24 ml blood was collected into EDTA-treated vacutainer tubes by venipuncture. Blood was immediately used for cell isolations as described below. All human subjects activities were approved by the Institutional Review Board at the University of Memphis under protocol 4316, and subjects provided informed consent prior to enrollment.

\section{Cell isolations}

Assays were performed on purified human classical monocytes isolated using immunomagnetic negative sorting (EasySep Direct Human Monocyte Isolation Kit, StemCell Technologies, Cambridge, 
MA). As we have previously described ${ }^{88}$, this procedure results in a highly pure (> $85 \%$ ) population of classical monocytes, with depletion of intermediate and non-classical monocytes due to the presence of an anti-CD16 antibody in the cocktail. Isolation purity was verified at several points throughout the current study and averaged approximately $90 \%$ (not shown). Cells were counted at $10 \times$ dilution using a Scepter cell counter (Millipore Sigma, St. Louis, MO). Isolated monocytes were immediately utilized in downstream assays, and no cells were frozen for later use.

\section{Media and reagents}

Unless otherwise specified, all assays were performed using Seahorse XF base DMEM medium (Agilent, Santa Clara, CA) supplemented with 10 mM glucose and 2 mM L-glutamine (Millipore Sigma, St. Louis, MO). Assays utilizing glucose deprivation omitted glucose from the media preparation. Media was not supplemented with fetal bovine serum or other additives. Recombinant spike protein subunit 1 (S1) was purchased from RayBiotech (Peachtree Corners, GA). 2-deoxyglucose, chetomin, compound C, and metformin were purchased from Millipore Sigma (St. Louis, MO). SARS-CoV-2 WA1/2020 strain was provided by Dr. Colleen Jonsson, Regional Biocontainment Laboratory, University of Tennessee Health Science Center.

\section{Seahorse extracellular flux}

Glycolysis and oxidative phosphorylation were respectively quantified via kinetic monitoring of extracellular acidification rate (ECAR) and oxygen consumption rate (OCR) on a Seahorse XFp analyzer (Agilent, Santa Clara, CA). For all assays, monocytes were plated at $1.5 \times 10^{5}$ cells per well, and wells A and $\mathrm{H}$ of the XFp plate were background wells with no cells. All analyses were run in duplicate. Plated cells were incubated at $37^{\circ} \mathrm{C}$ in a non- $\mathrm{CO}_{2}$ incubator for 1 hour prior to assays to stabilize $\mathrm{pH}$. All wells were imaged at $10 \times$ magnification for cell counting in order to adjust raw measurements for cell number. 
For quantification of dose response to $S 1,5$ basal measurements were made, followed by injection of media (wells B-C), $100 \mathrm{nM}$ spike protein (wells D-E), or $300 \mathrm{nM}$ spike protein (wells F-G). After injection into existing media in the well, spike protein concentrations were 10 -fold lower than injection concentrations, thereby giving final spike protein concentrations of $0 \mathrm{nM}, 10 \mathrm{nM}$, or $30 \mathrm{nM}$. Following injection, ECAR and OCR were monitored serially for 60 measurements. Following the assay, cell culture supernatants were removed, pooled by duplicate, and stored at $-80^{\circ} \mathrm{C}$. Cells were then lysed with $100 \mu \mathrm{l}$ Trizol (Thermo Fisher Scientific, Waltham, MA), pooled by duplicate, and stored at $-80^{\circ} \mathrm{C}$ as we have previously described ${ }^{89}$.

For chetomin and metformin Seahorse assays, cells were incubated in media as above (wells BE), or either $10 \mathrm{nM}$ chetomin or $50 \mathrm{mM}$ metformin during the 1-hour pre-incubation period (wells F-G). 5 basal ECAR/OCR measurements were performed, followed by injection of media (wells B-C) or $300 \mathrm{nM}$ spike protein (wells D-G) for a final concentration of $0 \mathrm{nM}$ (wells B-C) or $30 \mathrm{nM}$ spike protein (wells D-G) as above. Following injection, ECAR and OCR were monitored serially for 30 measurements. Cell culture supernatants and Trizol lysates were processed as described above following the end of the assay.

For glycolysis inhibition assays, cells were incubated in media, $10 \mathrm{mM}$ 2-deoxyglucose, or media without glucose (glucose deprivation) during the 1-hour pre-incubation period. 5 basal ECAR/OCR measurements were performed, followed by injection of $300 \mathrm{nM}$ spike protein to all wells for a final concentration of $30 \mathrm{nM}$ spike protein per well as above. Spike protein was prepared in non-glucose media for the glucose deprivation condition. Following injection, ECAR and OCR were monitored serially for 30 measurements. Cell culture supernatants and Trizol lysates were processed as described above following the end of the assay.

For glycolysis inhibition with compound C assays, cells were incubated in media, glucose deprivation media, or glucose deprivation media plus $10 \mu \mathrm{M}$ compound $\mathrm{C}$ during the 1-hour preincubation period. 5 basal ECAR/OCR measurements were performed, followed by injection of $300 \mathrm{nM}$ 
spike protein to all wells for a final concentration of $30 \mathrm{nM}$ spike protein per well as above. Spike protein was prepared in non-glucose media for the glucose deprivation condition. Following injection, ECAR and OCR were monitored serially for 30 measurements. Cell culture supernatants and Trizol lysates were processed as described above following the end of the assay.

\section{SARS-CoV-2 infections}

Isolated monocytes were incubated in RPMI-1640 media (Gibco, Thermo Fisher Scientific, Waltham, MA) supplemented with $10 \%$ fetal bovine serum (Gibco), with or without $50 \mathrm{mM}$ metformin, for 1-hour. Cells were then treated with media or infected with SARS-CoV-2 virus (WA1/2020 isolate) at $0.5 \mathrm{MOI}$ and incubated for 24 hours. Cell culture supernatants were collected from untreated and infected cells and stored at $-80^{\circ} \mathrm{C}$ until analysis.

\section{Gene and protein expression analysis}

RNA isolation was performed using the Trizol procedure based on manufacturer's instructions from cells lysed directly in the microplate or Seahorse plate wells as applicable. Isolated RNA (300-400 ng depending on experiment) was reverse-transcribed to cDNA using a High-Capacity cDNA Reverse Transcription Kit (Thermo Fisher Scientific, Waltham, MA). Gene expression was analyzed using commercial pre-validated gene expression assays and Taqman reagents (Thermo Fisher Scientific, Waltham, MA). Relative gene expression was quantified using the $2^{-\Delta \Delta C t}$ method ${ }^{90}$ against $B 2 M$ or $A C T B$ as housekeeping genes. Primer/probe IDs were: B2M Hs00187842_m1; ACTB Hs03023943_g1; IL1B Hs01555410_m1; IL6 Hs00174131_m1; CXCL8 Hs00174103_m1; TNF Hs00174128_m1.

For protein quantification, cell culture supernatants harvested from microplates or Seahorse XFp plates were analyzed via ELISA. Commercial DuoSet matched-antibody reagent sets were purchased from R\&D Systems (Minneapolis, MN) for quantifying human IL-6 and human TNF $\alpha$ and used according to manufacturer's instructions. All samples were run in duplicate at $5 \times$ dilution (SARS-CoV- 2 assays) or 50x dilution (Seahorse S1 dose response assays) and assessed against a standard curve. 
Protein concentration of angiotensin converting enzyme 2 (ACE2) and C-reactive protein (CRP) was performed by ELISA on plasma samples collected by venipuncture from subjects at the beginning of the study. Peripheral blood was collected by venipuncture into EDTA-coated vacutainer tubes, centrifuged at $1,500 \times \mathrm{g}$ for $15 \mathrm{~min}$, aliquoted, and stored at $-80^{\circ} \mathrm{C}$ until analysis. Plasma samples were analyzed in duplicate at $10 \times$ (ACE2) or 10,000x (CRP) using commercial DuoSet matched-antibody reagent kits (R\&D Systems) according to manufacturer's instructions and assessed against a standard curve.

\section{Data Processing and Statistical Analysis}

All data processing and statistical analyses were performed using R v. 3.6.2 ${ }^{91}$. Isolated monocytes from each subject were given all treatments for each experiment, so data were paired and analyzed using within-subjects designs. Data were checked for normality by Shapiro-Wilk test and analyzed by one-way repeated measures ANOVA (RM-ANOVA, for data which met the normality assumption) or Friedman's test (for data which did not meet the normality assumption). For analyses with significant main effects, post hoc mean separation was performed using pairwise paired T tests (for RM-ANOVA) or pairwise Wilcoxon signed-rank tests (for Friedman's tests) with p-value adjustment using the Holm-Bonferroni method ${ }^{92}$. Significance cutoff was $p<0.05$.

\section{Acknowledgements}

The authors would like to acknowledge the participants in this study. The authors thank Jyothi Parvathareddy and Colleen Jonsson from the Regional Biocontainment Laboratory at the University of Tennessee Health Science Center for providing SARS-CoV-2 virus stocks.

\section{Authors' Contributions}


BDP conceived the study. TJC and BDP designed experiments. TJC, RSE, JRY, KLD, and BDP collected data.

BDP analyzed data and prepared the first manuscript draft. TJC, RSE, JRY, KLD, and BDP edited the manuscript draft. All authors read and approved the final manuscript.

\section{Funding}

The study was primarily supported by a University of Memphis/University of Tennessee Health Science

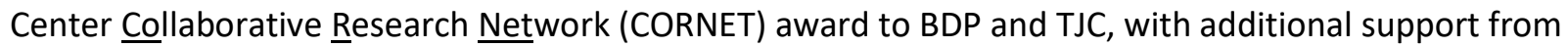
American Heart Association grants 18AIREA33961089 and 19TPA34910232 to BDP, and a University of Memphis College of Health Sciences faculty research grant to BDP. RSE was supported by a postdoctoral fellowship funded by the University of Memphis Division of Research and Innovation through the Carnegie R1 Postdoc Program. 


\section{References}

1. Worldometer. COVID-19 coronavirus pandemic. (2021). Available at:

https://www.worldometers.info/coronavirus/. (Accessed: 21st April 2021)

2. Gorbalenya, A. E. et al. The species Severe acute respiratory syndrome-related coronavirus:

classifying 2019-nCoV and naming it SARS-CoV-2. Nat. Microbiol. 5, 536-544 (2020).

3. Chen, G. et al. Clinical and immunologic features in severe and moderate Coronavirus Disease

2019. J. Clin. Invest. (2020). doi:10.1172/jci137244

4. Vangeti, S., Yu, M. \& Smed-Sörensen, A. Respiratory Mononuclear Phagocytes in Human

Influenza A Virus Infection: Their Role in Immune Protection and As Targets of the Virus. Front.

Immunol. 9, 1521 (2018).

5. Kimpen, J. L. L. Respiratory syncytial virus and asthma: The role of monocytes. Am. J. Respir. Crit.

Care Med. 163, S7-9 (2001).

6. Nikitina, E., Larionova, I., Choinzonov, E. \& Kzhyshkowska, J. Monocytes and macrophages as viral targets and reservoirs. Int. J. Mol. Sci. 19, 2821 (2018).

7. Pence, B. D. Severe COVID-19 and aging: are monocytes the key? GeroScience 42, 1051-1061

(2020).

8. Liao, M. et al. Single-cell landscape of bronchoalveolar immune cells in patients with COVID-19.

Nat. Med. 26, 842-844 (2020).

9. $\mathrm{Xu}, \mathrm{G}$. et al. The differential immune responses to COVID-19 in peripheral and lung revealed by single-cell RNA sequencing. Cell Discov. 6, 73 (2020).

10. Shaath, H., Vishnubalaji, R., Elkord, E. \& Alajez, N. M. Single-Cell Transcriptome Analysis Highlights a Role for Neutrophils and Inflammatory Macrophages in the Pathogenesis of Severe COVID-19. Cells 9, 2374 (2020).

11. Ren, X. et al. COVID-19 immune features revealed by a large-scale single cell transcriptome atlas. 
Cell (2021). doi:10.1016/j.cell.2021.01.053

12. Song, J. W. et al. Immunological and inflammatory profiles in mild and severe cases of COVID-19.

Nat. Commun. 11, 3410 (2020).

13. Nienhold, R. et al. Two distinct immunopathological profiles in autopsy lungs of COVID-19. Nat.

Commun. 11, 5086 (2020).

14. Beigmohammadi, M. T. et al. Pathological Findings of Postmortem Biopsies From Lung, Heart, and Liver of 7 Deceased COVID-19 Patients. Int. J. Surg. Pathol. (2020).

doi:10.1177/1066896920935195

15. Li, S. et al. Clinical and pathological investigation of patients with severe COVID-19. JCI Insight 5, e138070 (2020).

16. Winkler, E. S. et al. SARS-CoV-2 infection of human ACE2-transgenic mice causes severe lung inflammation and impaired function. Nat. Immunol. 21, 1327-1335 (2020).

17. Bao, L. et al. The pathogenicity of SARS-CoV-2 in hACE2 transgenic mice. Nature $\mathbf{5 8 3}, \mathbf{8 3 0 - 8 3 3}$ (2020).

18. Sia, S. F. et al. Pathogenesis and transmissio of SARS-CoV-2 in golden hamsters. Nature (2020). doi:10.1038/s41586-020-2342-5

19. Song, T. Z. et al. Delayed severe cytokine storm and immune cell infiltration in SARS-CoV-2infected aged Chinese rhesus macaques. Zool. Res. 41, 503-516 (2020).

20. Shan, C. et al. Infection with novel coronavirus (SARS-CoV-2) causes pneumonia in Rhesus macaques. Cell Res. 30, 670-677 (2020).

21. Chandrashekar, A. et al. SARS-CoV-2 infection protects against rechallenge in rhesus macaques.

Science 369, 812-817 (2020).

22. Speranza, E. et al. Single-cell RNA sequencing reveals SARS-CoV-2 infection dynamics in lungs of African green monkeys. Sci. Transl. Med. 13, eabe8146 (2021). 
23. Fahlberg, M. D. et al. Cellular events of acute, resolving or progressive COVID-19 in SARS-CoV-2 infected non-human primates. Nat. Commun. 11, 6078 (2020).

24. Giamarellos-Bourboulis, E. J. et al. Complex Immune Dysregulation in COVID-19 Patients with Severe Respiratory Failure. Cell Host Microbe 27, 992-1000.e3 (2020).

25. Laing, A. G. et al. A dynamic COVID-19 immune signature includes associations with poor prognosis. Nat. Med. 26, 1623-1635 (2020).

26. Schulte-Schrepping, J. et al. Severe COVID-19 Is Marked by a Dysregulated Myeloid Cell Compartment. Cell 182, 1419-1440.e23 (2020).

27. Payen, D. et al. A Longitudinal Study of Immune Cells in Severe COVID-19 Patients. Front. Immunol. 11, 580250 (2020).

28. Mann, E. R. et al. Longitudinal immune profiling reveals key myeloid signatures associated with COVID-19. Sci. Immunol. 5, eabd6197 (2020).

29. Kvedaraite, E. et al. Major alterations in the mononuclear phagocyte landscape associated with COVID-19 severity. Proc. Natl. Acad. Sci. 118, e2018587118 (2021).

30. Varchetta, S. et al. Unique immunological profile in patients with COVID-19. Cell. Mol. Immunol. (2020). doi:10.1038/s41423-020-00557-9

31. Su, Y. et al. Multi-Omics Resolves a Sharp Disease-State Shift between Mild and Moderate COVID19. Cell 183, 1479-1495.e20 (2020).

32. Guo, C. et al. Single-cell analysis of two severe COVID-19 patients reveals a monocyte-associated and tocilizumab-responding cytokine storm. Nat. Commun. 11, 3924 (2020).

33. Kahn, R. et al. Mismatch between circulating cytokines and spontaneous cytokine production by leukocytes in hyperinflammatory COVID-19. J. Leukoc. Biol. 109, 115-120 (2021).

34. Codo, A. C. et al. Elevated Glucose Levels Favor SARS-CoV-2 Infection and Monocyte Response through a HIF-1 $\alpha$ /Glycolysis-Dependent Axis. Cell Metab. 32, 437-446.e5 (2020). 
35. da Silva Gomes Dias, S. et al. Lipid droplets fuel SARS-CoV-2 replication and production of inflammatory mediators. PLoS Pathog. 16, e1009127 (2020).

36. Ferreira, A. C. et al. SARS-CoV-2 engages inflammasome and pyroptosis in human primary monocytes. Cell Death Discov. 7, 43 (2021).

37. Zheng, J. et al. Severe Acute Respiratory Syndrome Coronavirus 2-Induced Immune Activation and Death of Monocyte-Derived Human Macrophages and Dendritic Cells. J. Infect. Dis. jiaa753 (2020). doi:10.1093/infdis/jiaa753

38. Yang, D. et al. Attenuated interferon and proinflammatory response in SARS-CoV-2-infected human dendritic cells is associated with viral antagonism of STAT1 phosphorylation. J. Infect. Dis.

222, 734-745 (2020).

39. O'Neill, L. A. J., Kishton, R. J. \& Rathmell, J. A guide to immunometabolism for immunologists. Nat. Rev. Immunol. 16, 553-565 (2016).

40. Wang, W. et al. Up-regulation of IL- 6 and TNF- $\alpha$ induced by SARS-coronavirus spike protein in murine macrophages via NF-kB pathway. Virus Res. 128, 1-8 (2007).

41. Dosch, S. F., Mahajan, S. D. \& Collins, A. R. SARS coronavirus spike protein-induced innate immune response occurs via activation of the NF-KB pathway in human monocyte macrophages in vitro. Virus Res. 142, 19-27 (2009).

42. Ropa, J., Cooper, S., Capitano, M. L., Van't Hof, W. \& Broxmeyer, H. E. Human Hematopoietic Stem, Progenitor, and Immune Cells Respond Ex Vivo to SARS-CoV-2 Spike Protein. Stem Cell Rev. Reports (2020). doi:10.1007/s12015-020-10056-z

43. Karwaciak, I., Sałkowska, A., Karaś, K., Dastych, J. \& Ratajewski, M. Nucleocapsid and Spike Proteins of the Coronavirus SARS-CoV-2 Induce IL6 in Monocytes and Macrophages-Potential Implications for Cytokine Storm Syndrome. Vaccines 9, 54 (2021).

44. Thépaut, M. et al. DC/L-SIGN recognition of spike glycoprotein promotes SARS-CoV-2 trans- 
infection and can be inhibited by a glycomimetic antagonist. PLoS Pathog. 17, e1009576 (2021).

45. Lu, Q. et al. SARS-CoV-2 exacerbates proinflammatory responses in myeloid cells through C-type lectin receptors and Tweety family member 2. Immunity (2021).

46. Rodrigues, T. S. et al. Inflammasomes are activated in response to SARS-cov-2 infection and are associated with COVID-19 severity in patients. J. Exp. Med. 218, e20201707 (2020).

47. Cauchois, R. et al. Early IL-1 receptor blockade in severe inflammatory respiratory failure complicating COVID-19. Proc. Natl. Acad. Sci. U. S. A. 117, 18951-18953 (2020).

48. Lucas, C. et al. Longitudinal analyses reveal immunological misfiring in severe COVID-19. Nature 584, 463-469 (2020).

49. Blanco-Melo, D. et al. Imbalanced Host Response to SARS-CoV-2 Drives Development of COVID19. Cell 181, 1036-1045.e9 (2020).

50. Del Valle, D. M. et al. An inflammatory cytokine signature predicts COVID-19 severity and survival. Nat. Med. 26, 1636-1643 (2020).

51. Chen, L. et al. Scoring cytokine storm by the levels of MCP-3 and IL-8 accurately distinguished COVID-19 patients with high mortality. Signal Transduct. Target. Ther. 5, 292 (2020).

52. Cramer, T. et al. HIF-1 $\alpha$ is essential for myeloid cell-mediated inflammation. Cell 112, 645-657 (2003).

53. Tannahill, G. M. et al. Succinate is an inflammatory signal that induces IL-1 beta through HIF-1 alpha. Nature 496, 238-+ (2013).

54. Rius, J. et al. NF-kB links innate immunity to the hypoxic response through transcriptional regulation of HIF-1 $\alpha$. Nature 453, 807-811 (2008).

55. Corcoran, S. E. \& O'Neill, L. A. J. HIF1 $\alpha$ and metabolic reprogramming in inflammation. J. Clin. Invest. 126, 3699-3707 (2016).

56. Kung, A. L. et al. Small molecule blockade of transcriptional coactivation of the hypoxia-inducible 
factor pathway. Cancer Cell 6, 33-43 (2004).

57. Raulien, N. et al. Fatty acid oxidation compensates for lipopolysaccharide-induced Warburg effect in glucose-deprived monocytes. Front. Immunol. 8, 609 (2017).

58. Yarbro, J. R. \& Pence, B. D. Classical monocytes from older adults maintain capacity for metabolic compensation during glucose deprivation and lipopolysaccharide stimulation. Mech. Ageing Dev. 183, 111146 (2019).

59. Otto, N. A. et al. Adherence Affects Monocyte Innate Immune Function and Metabolic Reprogramming after Lipopolysaccharide Stimulation In Vitro. J. Immunol. 206, 827-838 (2021).

60. Woods, P. S. et al. Tissue-resident alveolar macrophages do not rely on glycolysis for LPS-induced inflammation. Am. J. Respir. Cell Mol. Biol. 62, 243-255 (2020).

61. Zhou, G. et al. Role of AMP-activated protein kinase in mechanism of metformin action. J. Clin. Invest. 108, 1167-1174 (2001).

62. Pajak, B. et al. 2-Deoxy-D-Glucose and its analogs: From diagnostic to therapeutic agents. Int. J. Mol. Sci. 21, 234 (2020).

63. Takiyama, Y. et al. Tubular injury in a rat model of type 2 diabetes is prevented by metformin: A possible role of HIF-1 $\alpha$ expression and oxygen metabolism. Diabetes 60, 981-992 (2011).

64. Zhou, X. et al. Metformin suppresses hypoxia-induced stabilization of HIF-1' through reprogramming of oxygen metabolism in hepatocellular carcinoma. Oncotarget 7, 873-884 (2016).

65. El-Mir, M. Y. et al. Dimethylbiguanide inhibits cell respiration via an indirect effect targeted on the respiratory chain complex I. J. Biol. Chem. 275, 223-228 (2000).

66. Owen, M. R., Doran, E. \& Halestrap, A. P. Evidence that metformin exerts its anti-diabetic effects through inhibition of complex 1 of the mitochondrial respiratory chain. Biochem. J. 348, 607-614 (2000). 
67. Zheng, M. et al. TLR2 senses the SARS-CoV-2 envelope protein to produce inflammatory cytokines. Nat. Immunol. (2021).

68. Fu, Y. Z. et al. SARS-CoV-2 membrane glycoprotein M antagonizes the MAVS-mediated innate antiviral response. Cell. Mol. Immunol. 18, 613-620 (2021).

69. Boumaza, A. et al. Monocytes and macrophages, targets of SARS-CoV-2: the clue for Covid-19 immunoparalysis. J. Infect. Dis. (2021).

70. Ogata, A. F. et al. Circulating SARS-CoV-2 Vaccine Antigen Detected in the Plasma of mRNA-1273 Vaccine Recipients. Clin. Infect. Dis. (2021).

71. Johnson, B. A. et al. Loss of furin cleavage site attenuates SARS-CoV-2 pathogenesis. Nature 591, 293-299 (2021).

72. Letarov, A. V., Babenko, V. V. \& Kulikov, E. E. Free SARS-CoV-2 Spike Protein S1 Particles May Play a Role in the Pathogenesis of COVID-19 Infection. Biochem. 86, 257-261 (2021).

73. Radzikowska, U. et al. Distribution of ACE2, CD147, CD26, and other SARS-CoV-2 associated molecules in tissues and immune cells in health and in asthma, COPD, obesity, hypertension, and COVID-19 risk factors. Allergy Eur. J. Allergy Clin. Immunol. 75, 2829-2845 (2020).

74. Wang, K. et al. CD147-spike protein is a novel route for SARS-CoV-2 infection to host cells. Signal Transduct. Target. Ther. 5, 283 (2020).

75. Hill, A. M., Barber, M. J. \& Gotham, D. Estimated costs of production and potential prices for the WHO essential medicines list. BMJ Glob. Heal. 3, (2018).

76. Science, J. M. E. C. for C. D. and C. in Comparative Effectiveness Review Summary Guides for Consumers (Agency for Healthcare Research and Quality, 2011).

77. Yu, L. L. et al. Metformin relieves acute respiratory distress syndrome by reducing miR-138 expression. Eur. Rev. Med. Pharmacol. Sci. 22, 5355-5363 (2018).

78. Kim, J. et al. Metformin suppresses lipopolysaccharide (LPS)-induced inflammatory response in 
murine macrophages via Activating Transcription Factor-3 (ATF-3) induction. J. Biol. Chem. 289, $23246-23255$ (2014).

79. Kelly, B., Tannahill, G. M., Murphy, M. P. \& O’Neill, L. A. J. Metformin inhibits the production of reactive oxygen species from $\mathrm{NADH}$ : Ubiquinone oxidoreductase to limit induction of interleukin$1 \beta$ (IL-1 $\beta$ ) and boosts interleukin-10 (IL-10) in lipopolysaccharide (LPS)-activated macrophages. J. Biol. Chem. 290, 20348-20359 (2015).

80. Arai, M. et al. Metformin, an antidiabetic agent, suppresses the production of tumor necrosis factor and tissue factor by inhibiting early growth response factor-1 expression in human monocytes in vitro. J. Pharmacol. Exp. Ther. 334, 206-213 (2010).

81. Crouse, A. B. et al. Metformin Use Is Associated With Reduced Mortality in a Diverse Population With COVID-19 and Diabetes. Front. Endocrinol. (Lausanne). 600439 (2021). doi:10.3389/fendo.2020.600439

82. Bramante, C. T. et al. Metformin and risk of mortality in patients hospitalised with COVID-19: a retrospective cohort analysis. Lancet Heal. Longev. 2, e34-e41 (2021).

83. Cariou, B. et al. Phenotypic characteristics and prognosis of inpatients with COVID-19 and diabetes: the CORONADO study. Diabetologia 63, 1500-1515 (2020).

84. Luo, P. et al. Metformin treatment was associated with decreased mortality in COVID-19 patients with diabetes in a retrospective analysis. Am. J. Trop. Med. Hyg. 103, 69-72 (2020).

85. Hariyanto, T. I. \& Kurniawan, A. Metformin use is associated with reduced mortality rate from coronavirus disease 2019 (COVID-19) infection. Obes. Med. 19, 100290 (2020).

86. Chen, Y. et al. Clinical Characteristics and Outcomes of Patients with Diabetes and COVID-19 in Association with Glucose-Lowering Medication. Diabetes Care 43, 1399-1407 (2020).

87. Cheng, X. et al. Metformin Is Associated with Higher Incidence of Acidosis, but Not Mortality, in Individuals with COVID-19 and Pre-existing Type 2 Diabetes. Cell Metab. 32, 537-547.e3 (2020). 
88. Pence, B. D. \& Yarbro, J. R. Aging impairs mitochondrial respiratory capacity in classical monocytes. Exp. Gerontol. 108, 112-117 (2018).

89. Pence, B. D. \& Yarbro, J. R. Classical monocytes maintain ex vivo glycolytic metabolism and early but not later inflammatory responses in older adults. Immun. Ageing 16, 3 (2019).

90. Livak, K. J. \& Schmittgen, T. D. Analysis of relative gene expression data using real-time quantitative PCR and the 2(-Delta Delta C(T)) Method. Methods 25, 402-408 (2001).

91. R Core Team. R: A language and environment for statistical computing. R Foundation for Statistical Computing, Vienna, Austria. (2019).

92. Sture Holm. A Simple Sequentially Rejective Multiple Test Procedure. Scand. J. Stat. 6, 65-70 (1979). 


\section{Figure Legends}

Figure 1. Recombinant SARS-CoV-2 spike protein subunit 1 ( $\mathrm{rS} 1)$ mediates immunometabolic activation of monocytes. (A) Monocytes increase extracellular acidification response rate (ECAR) in a dosedependent manner when treated with rS1. (B) Quantification of ECAR by area under the curve (AUC). (C) rS1 treatment suppresses oxygen consumption rate (OCR) in monocytes in a dose-dependent fashion. (D) Quantification of OCR by AUC. (E) Gene expression analysis by qPCR reveals dose-dependent increases in responses of IL1B, IL6, CXCL8, and TNF to rS1 stimulation. (F) Protein expression analysis by ELISA reveals dose-dependent increases in responses of IL-6 and TNFa to rS1 stimulation. ECAR and OCR data in panels A-D are adjusted for values indexed to $1 \times 10^{5}$ cells/well. * ,**,***: $p<0.05, p<0.01$, p<0.001 vs. $0 \mathrm{nM} \mathrm{rS1.}+,+\dagger,++\dagger: p<0.05, p<0.01, p<0.001$ vs. $10 \mathrm{nM} \mathrm{rS1.} \mathrm{N}=8$ biological replicates.

Figure 2. HIF-1 $\alpha$ inhibition suppresses immunometabolic activation of monocytes due to recombinant spike protein (rS1). (A) Monocytes increase extracellular acidification response rate (ECAR) when treated with $30 \mathrm{nM} \mathrm{rS1}$, but this is blocked by pre-treatment with chetomin. (B) Quantification of ECAR by area under the curve (AUC). (C) rS1 increase of expression of IL1B, IL6, CXCL8, and TNF is reversed by chetomin pre-treatment. ${ }^{*}, * *, * * *: p<0.05, p<0.01, p<0.001$ vs. untreated cells. $\dagger^{+},+,+\dagger+: p<0.05$, $\mathrm{p}<0.01, \mathrm{p}<0.001$ vs. rS1-treated cells. $\mathrm{N}=7$ biological replicates.

Figure 3. Targeting glycolysis has variable effects on recombinant spike protein (rS1) responses in monocytes. (A) Glucose deprivation or pre-treatment with 2-deoxyglucose (2-DG) block extracellular acidification rate (ECAR) increase due to $\mathrm{rS1}$ treatment. (B) Quantification of ECAR by area under the curve (AUC). (C) 2-DG inhibits oxygen consumption rate in rS1-treated monocytes, but glucose-deprived monocytes upregulate OCR in response to rS1. (D) Quantification of OCR by AUC. (E) 2-DG blocks 
expression of ILIB, IL6, CXCL8, and TNF due to rS1 stimulation, but glucose deprivation has limited effects on cytokine expression. ${ }^{*}, * *, * * *: p<0.05, p<0.01, p<0.001$ vs. rS1-treated cells. ${ }^{*},+\dagger,+\dagger+$ : $p<0.05, p<0.01, p<0.001$ vs. 2-DG-treated cells. $N=7$ biological replicates.

Figure 4. Inhibiting AMPK blocks metabolic response to recombinant spike protein subunit 1 (rS1) in glucose-deprived monocytes but has minimal effect on most cytokines. (A) Compound C pre-treatment has no effect on extracellular acidification (ECAR). (B) Quantification of ECAR by area under the curve (AUC). (C) Compound C blocks the increase in oxygen consumption rate (OCR) in rS1-treated glucosedeprived monocytes. (D) Quantification of OCR by AUC. (E) Compound C pre-treatment inhibits TNF expression in glucose-deprived monocytes stimulated with rS1, but does not significantly alter expression of IL1B, IL6, or CXCL8. * ,**,***: p<0.05, p<0.01, p<0.001 vs. glucose-treated cells. $\dagger^{*},+, \dagger^{+}+$: $p<0.05, p<0.01, p<0.001$ vs. glucose-deprived cells. $N=7$ biological replicates.

Figure 5. Metformin suppresses immunometabolic activation in monocytes treated with recombinant spike protein (rS1). (A) Metformin pre-treatment blocks the increase in extracellular acidification rate (ECAR) mediated by rS1. (B) Quantification of ECAR by area under the curve (AUC). (C) Metformin suppresses oxygen consumption rate (OCR). (D) Quantification of OCR by AUC. (E) Metformin suppresses cytokine responses, as demonstrated by gene expression of IL1B, IL6, CXCL8, and TNF, during rS1 stimulation in monocytes. ${ }^{*}, * *, * * *: p<0.05, p<0.01, p<0.001$ vs. unstimulated cells. $\dagger^{+}+\dagger,+\dagger+: p<0.05$, $\mathrm{p}<0.01, \mathrm{p}<0.001$ vs. rS1-treated cells. $\mathrm{N}=7$ biological replicates. 
bioRxiv preprint doi: https://doi.org/10.1101/2021.05.27.445991; this version posted May 28, 2021. The copyright holder for this preprint (which

was not certified by peer review) is the author/funder, who has granted bioRxiv a license to display the preprint in perpetuity. It is made available under aCC-BY 4.0 International license.

Figure 6. Metformin inhibits IL-6 production in monocytes infected with SARS-CoV-2 strain WA1/2020

(Cov2) at 0.5 MOI. *: $p<0.05$ vs. uninfected cells. + : $p<0.05$ vs. Cov2-infected cells. $\mathrm{N}=8$ biological replicates. 

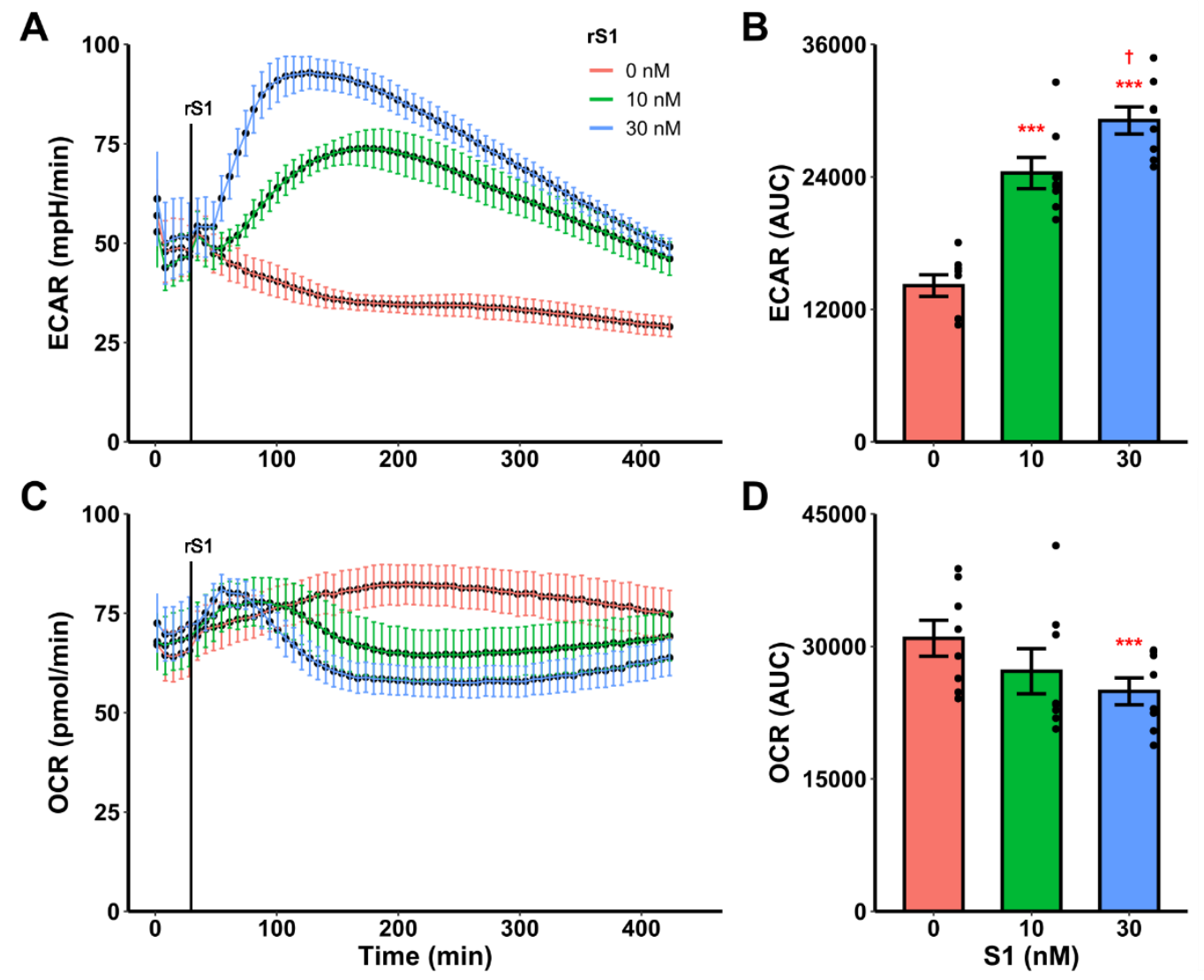

E

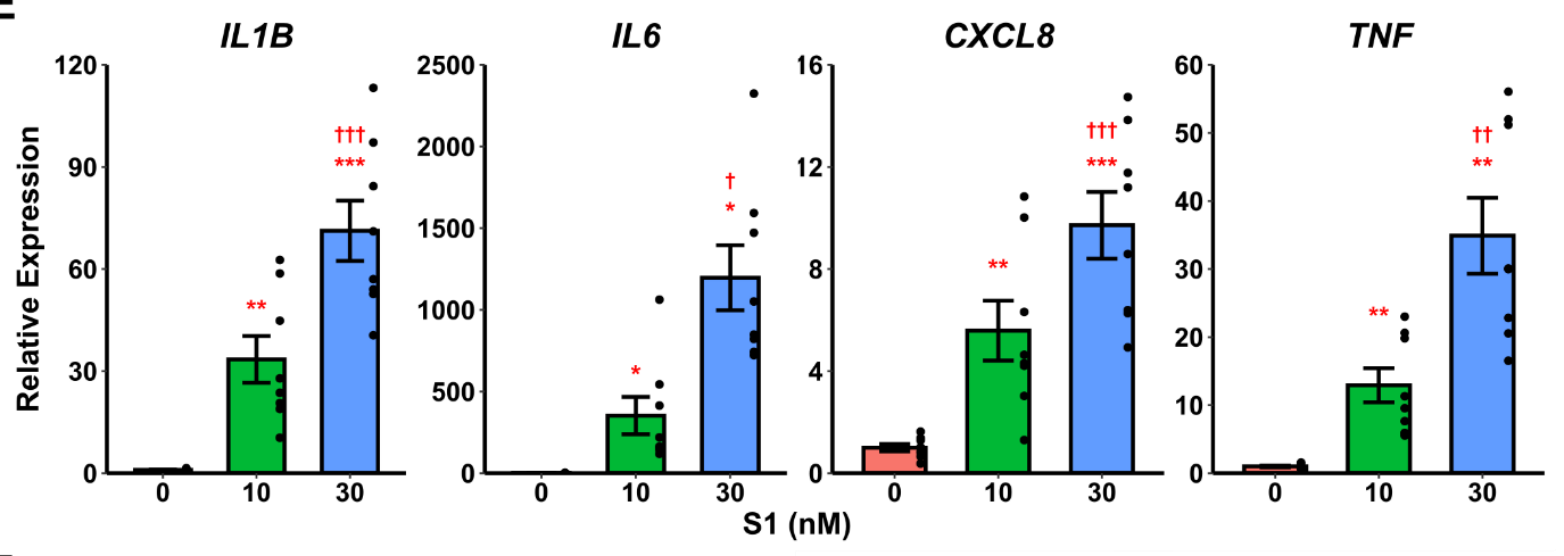

F

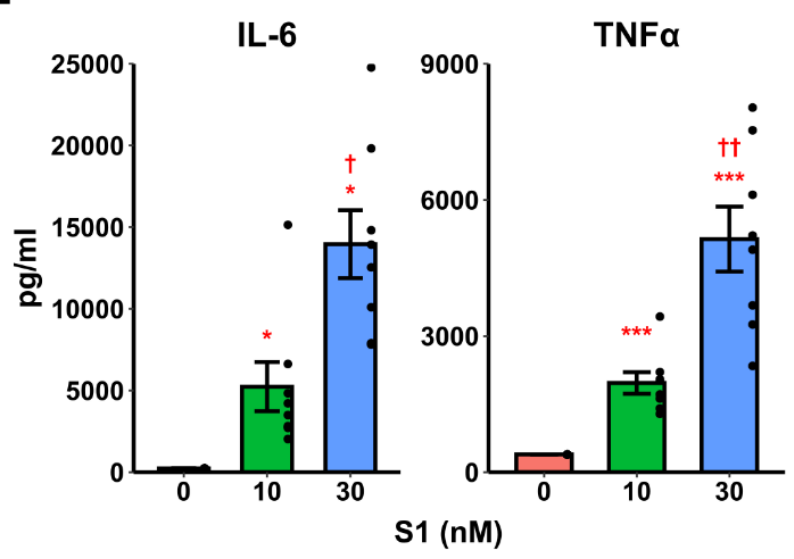

(Fig 1)

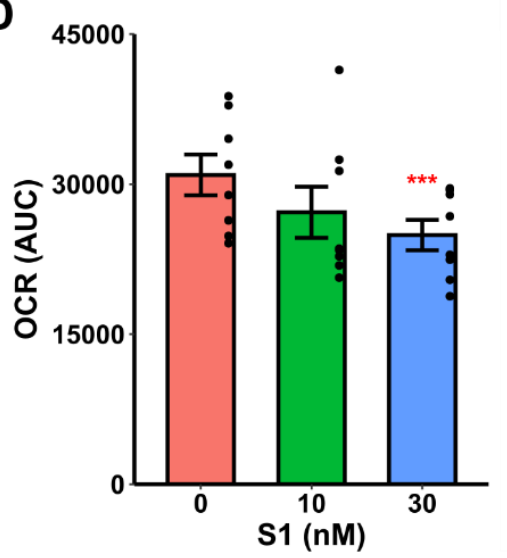



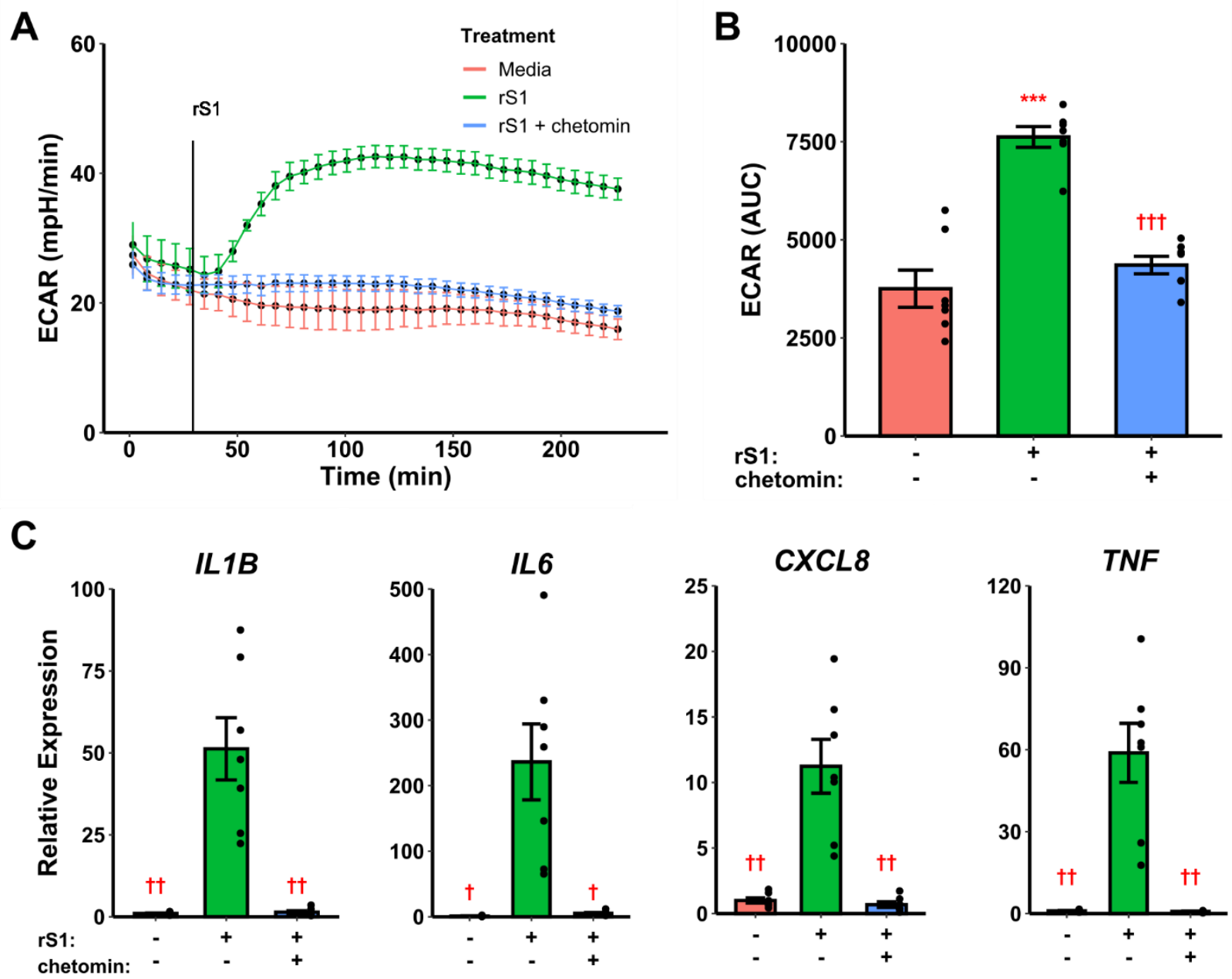

(Fig 2) 

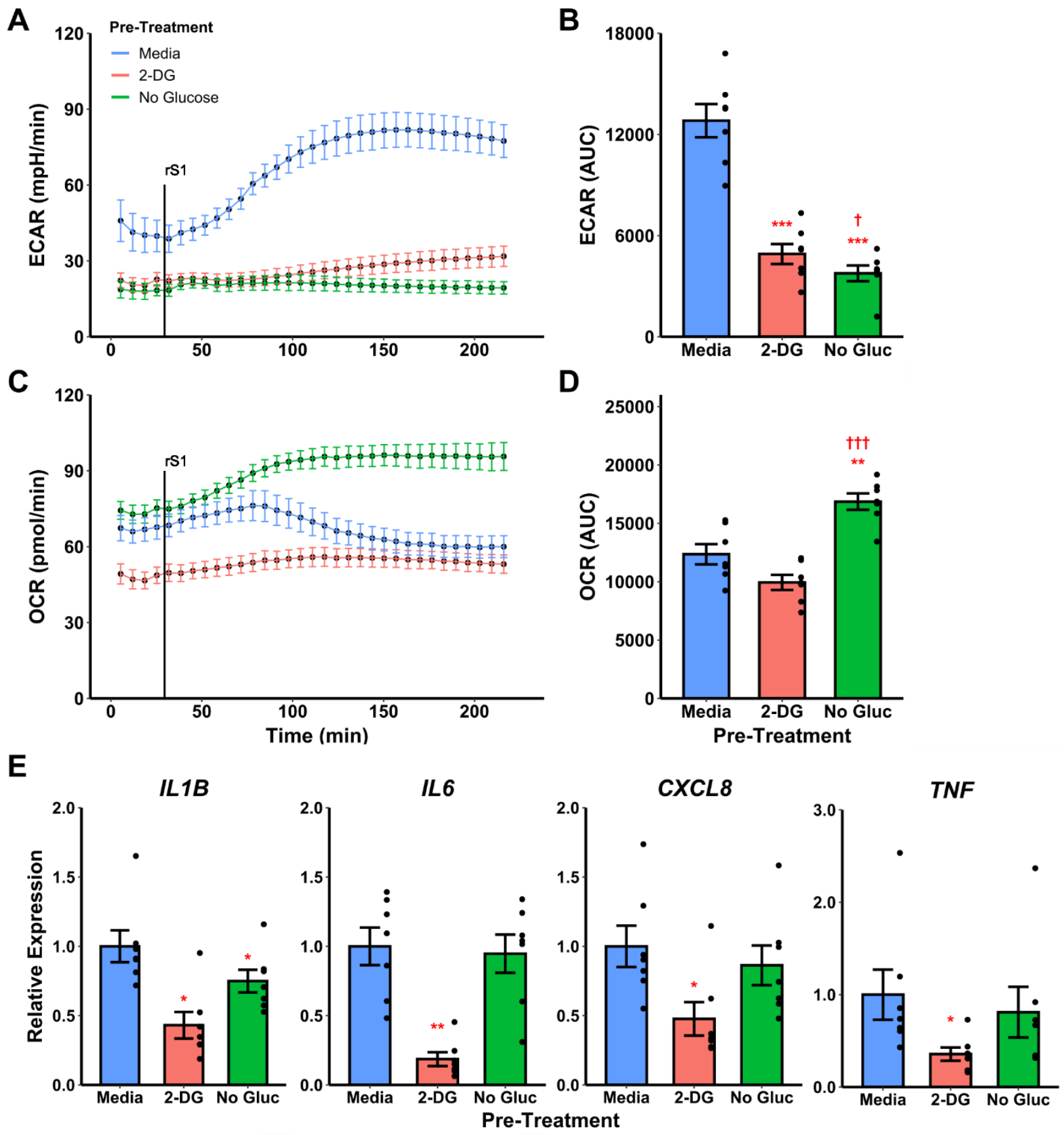

(Fig 3) 

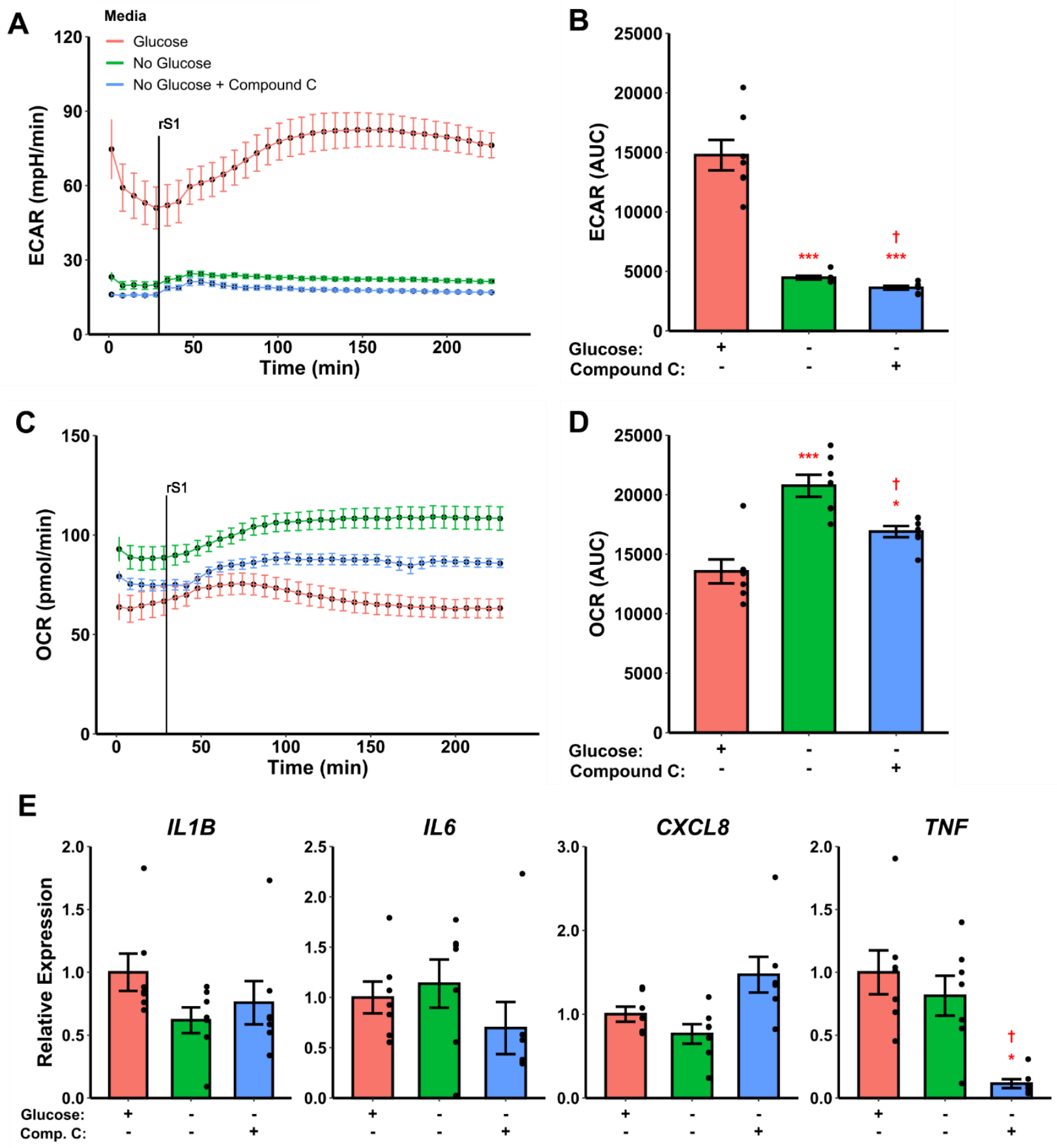

(Fig 4) 

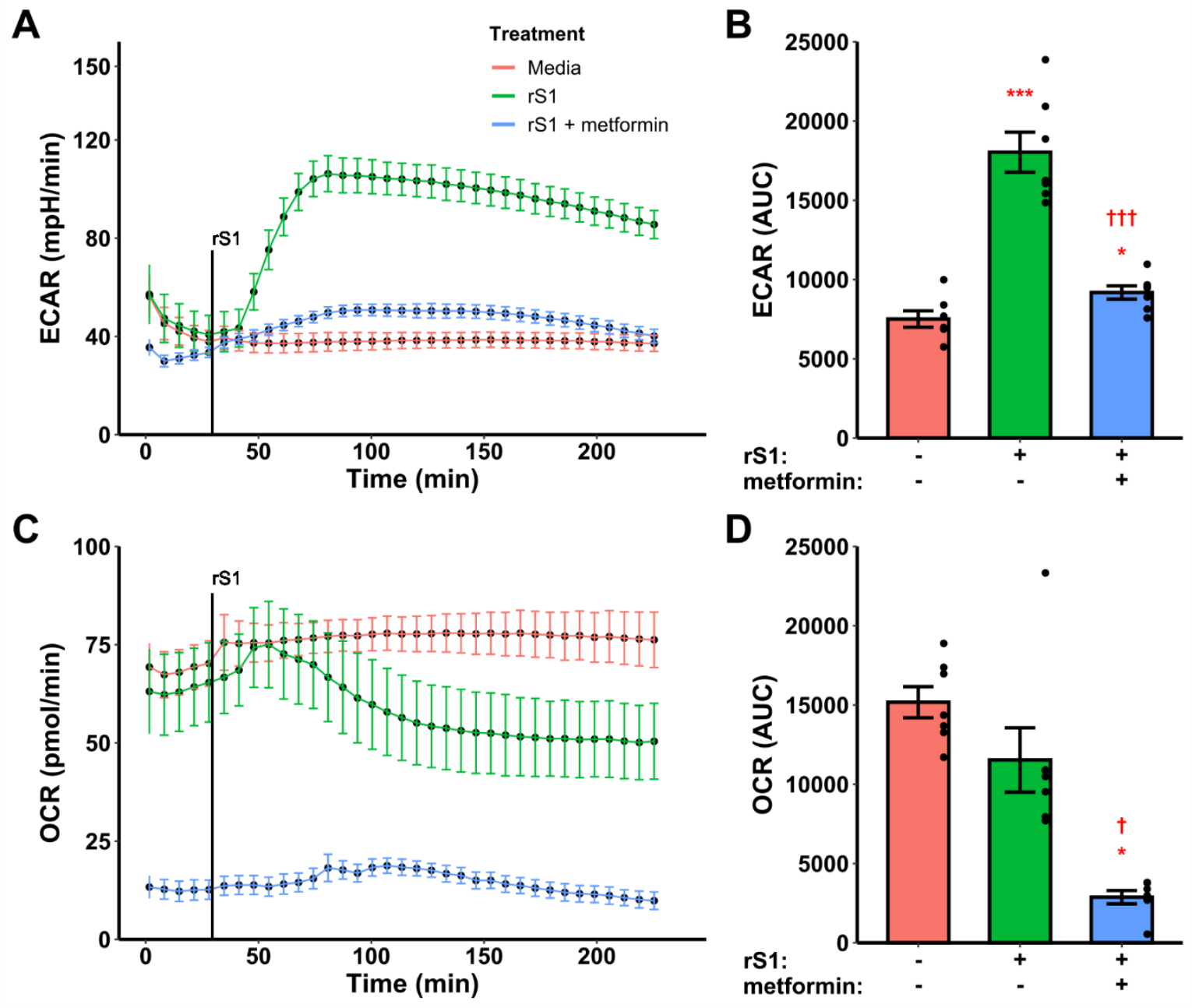

E
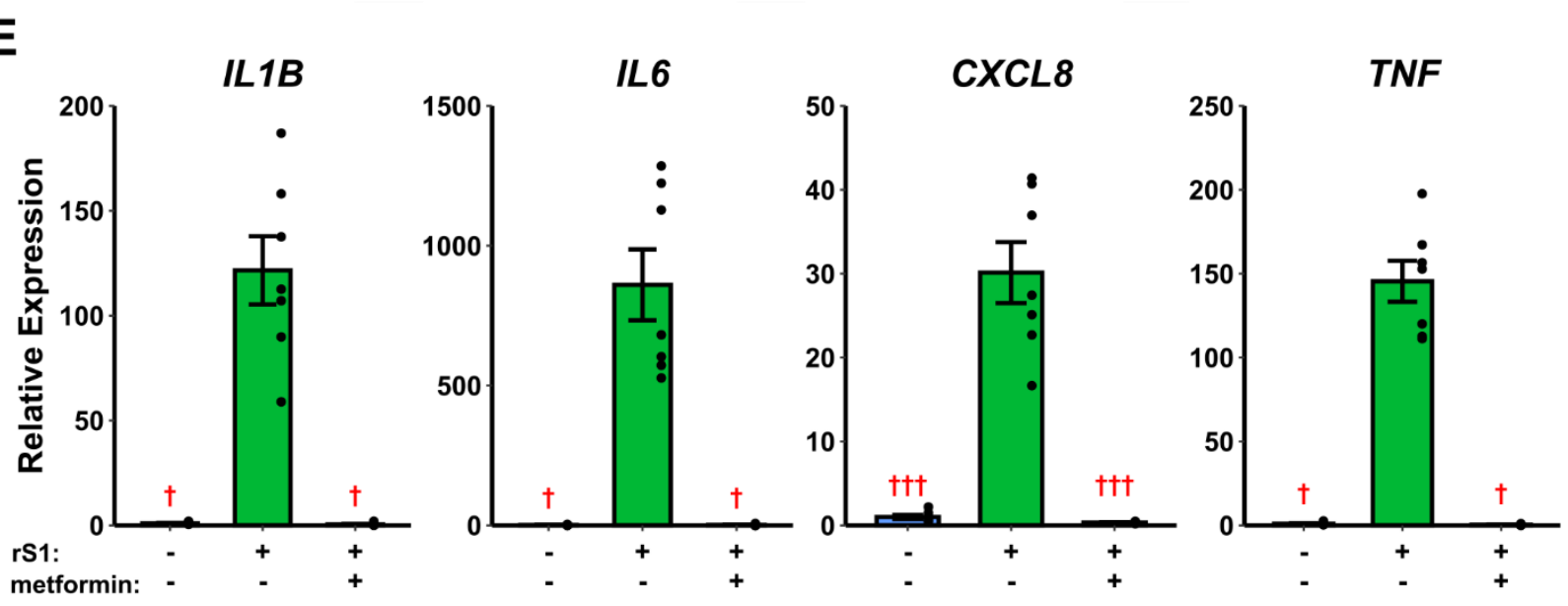

(Fig 5) 
bioRxiv preprint doi: https://doi.org/10.1101/2021.05.27.445991; this version posted May 28, 2021. The copyright holder for this preprint (which

was not certified by peer review) is the author/funder, who has granted bioRxiv a license to display the preprint in perpetuity. It is made available under aCC-BY 4.0 International license.

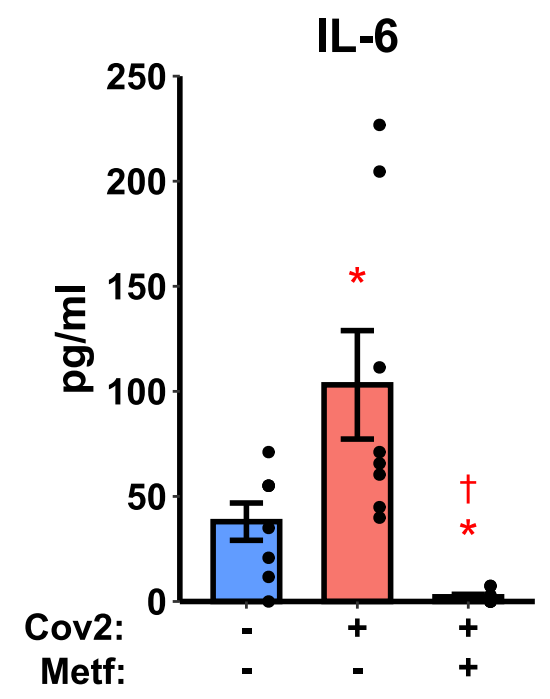

(Fig 6) 